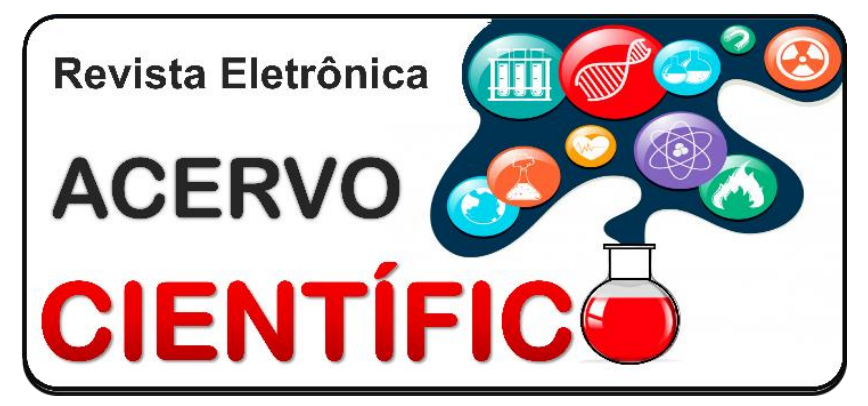

REVISÃO BIBLIOGRÁFICA

Recebido em: 7/2020

Aceito em: $8 / 2020$

Publicado em: 10/2020

\title{
Os limites entre a episiotomia de rotina e a violência obstétrica
}

\author{
The limits between a routine episiotomy and obstetric violence \\ Los limites entre una episiotomía rutinaria y violencia obstétrica
}

Marcela Távora de Freitas ${ }^{1 *}$, Danielle Fiorin Ferrari Novais ${ }^{2}$, Alice Crespo Brito ${ }^{3}$, Camila Carolina Canedo Campos ${ }^{1}$, Clara Dinalli Ornellas Iglesias ${ }^{4}$, Larissa Griffo Gonçalves ${ }^{1}$, Leticia Uhlig Grosman $^{3}$, Maressa Melo Oliveira ${ }^{3}$, Thays Sturzeneker de Alcantara ${ }^{1}$, Vitória Lopes Dornelas de Carvalho'.

Resumo: Este artigo buscou realizar uma revisão a respeito de qual momento a episiotomia deixa de ser benéfica e se torna uma violência obstétrica. Sabe-se que durante o parto natural é necessário que ocorra uma expansão vaginal e cervical de forma lenta, permitindo, assim, a passagem do feto. Porém, muitas vezes, na justificativa de facilitar o nascimento e prevenir lesões perineais, as mulheres são submetidas a uma incisão cirúrgica na região do períneo, processo denominado episiotomia. A recomendação da Organização Mundial de Saúde é que o índice de realização dessa intervenção não ultrapasse $30 \%$, todavia no Brasil esse número é superior ao proposto. Tal procedimento resulta em impactos negativos à mulher, tanto físicos quanto psicológicos, tais como incontinência urinária e fecal, dispareunia, sentimentos de vergonha e medo. Além disso, muitas gestantes não são informadas previamente sobre o procedimento a ser realizado, ou seja, seus direitos e autonomia são violados. Portanto, é necessário que as gestantes sejam informadas durante o pré-natal sobre as possíveis intervenções que possam ocorrer durante 0 processo de parto e, ademais, que os profissionais de saúde realizem a episiotomia apenas em casos seletivos, visando à humanização do parto.

Palavras chave: Episiotomia, Obstetrícia, Violência contra a mulher.

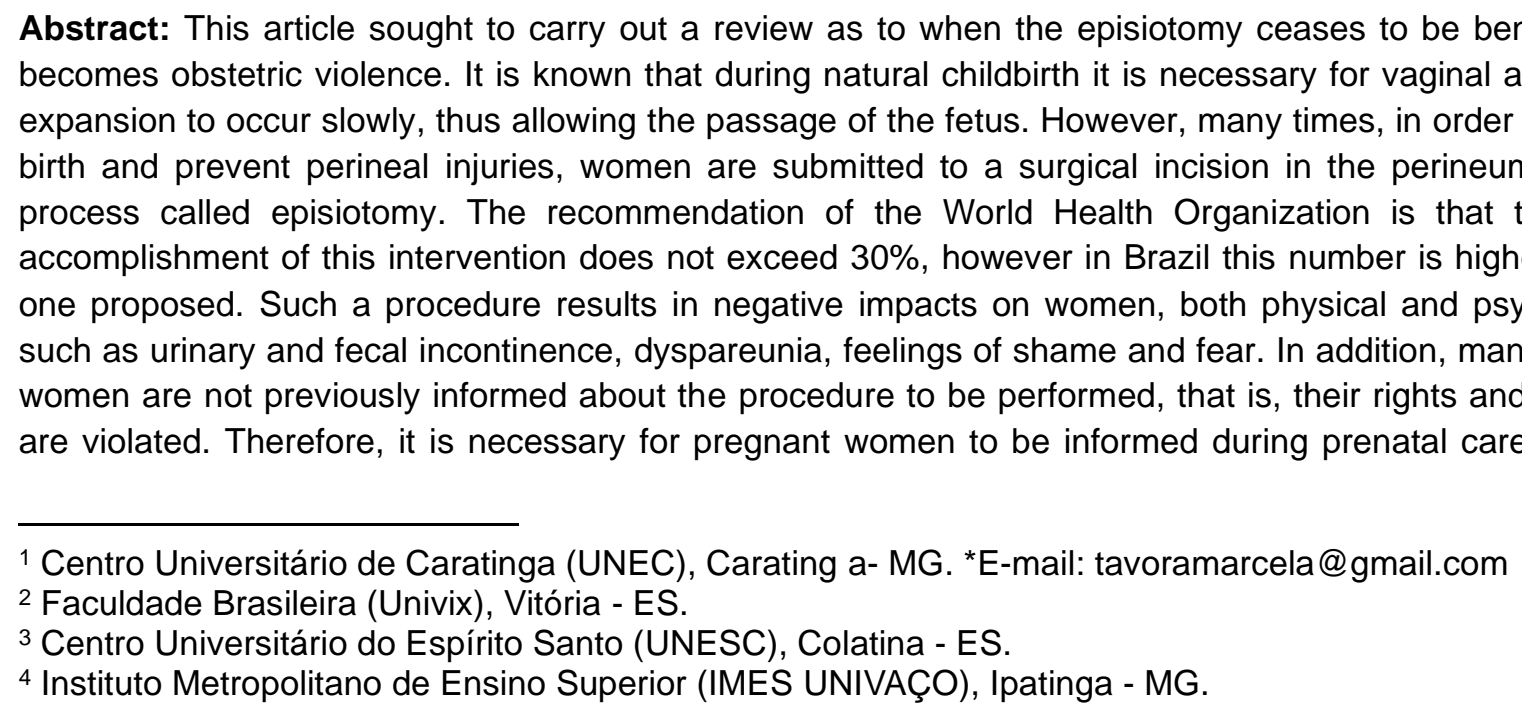

REAC/EJSC | Vol. 13 | e4696 | DOI: https://doi.org/10.25248/reac.e4696.2020

Página 1 de 7 
possible interventions that may occur during the delivery process and, in addition, that health professionals perform episiotomy only in selective cases, aiming at the humanization of delivery.

Keywords: Episiotomy, Obstetrics, Violence against women.

Resumen: Este artículo buscó realizar una revisión sobre cuándo la episiotomía deja de ser beneficiosa y se convierte en violencia obstétrica. Se sabe que durante el parto natural es necesario que la expansión vaginal y cervical se produzca lentamente, permitiendo así el paso del feto. Sin embargo, muchas veces, para facilitar el parto y prevenir lesiones perineales, las mujeres se someten a una incisión quirúrgica en la región del perineo, un proceso llamado episiotomía. La recomendación de la Organización Mundial de la Salud es que la tasa de realización de esta intervención no supere el $30 \%$, sin embargo en Brasil este número es superior al propuesto. Tal procedimiento tiene como resultado impactos negativos en la mujer, tanto físicos como psicológicos, como incontinencia urinaria y fecal, dispareunia, sentimientos de vergüenza y miedo. Además, muchas mujeres embarazadas no son informadas previamente sobre el procedimiento a realizar, es decir, se vulneran sus derechos y autonomía. Por ello, es necesario que la gestante esté informada durante el prenatal sobre las posibles intervenciones que pueden ocurrir durante el proceso del parto y, además, que los profesionales de la salud realicen la episiotomía solo en casos selectivos, con el objetivo de humanizar el parto.

Palabras clave: Episiotomía, Obstetricia, Violencia contra la mujer.

\section{INTRODUÇÃO}

O nascimento é um episódio fisiológico que envolve inúmeras interpretações culturais (CARNIEL F, et al., 2019). O parto vaginal é um processo que, para ocorrer de modo adequado, depende que a expansão vaginal e cervical aconteça apropriadamente para a passagem do feto (GÜN I, et al., 2016). A fim de obter a expansão perineal, principalmente em mulheres primíparas, muitos médicos obstetras optam por realizar uma incisão cirúrgica denominada episiotomia (DENGO VAR, 2016), a qual foi criada no ano de 1742 por Fielding Ould, que defendeu seu uso apenas em situações específicas, em que houvessem dificuldades nos partos (GARRETT CA, et al., 2016).

Entretanto, essa técnica foi amplamente inserida na prática médica após uma publicação em 1920 de DeLee, médico obstetra que defendeu o uso rotineiro da episiotomia. Tal progressão no uso da técnica ocorreu até o ano de 1983, ocasião em que dois epidemiologistas publicaram uma revisão expondo que não havia evidências concretas de benefícios ou dos riscos da episiotomia (JUNIOR MD e JÚNIOR RP, 2016). A partir disso, mundialmente, houve um crescente número de pesquisas relacionadas a esse procedimento em todo o mundo e, com isso, no ano de 2009 a Cochrane Library publicou um estudo baseado em metanálise no qual concluiu-se que a episiotomia era mais indicada em casos seletivos do que em rotineiros (CARROLI G e MIGNINI L, 2009).

Em virtude das novas indicações da prática da episiotomia, a Organização Mundial da Saúde (OMS) recomenda que, nos partos normais, o índice de realização dessa incisão deva ser de $15 \%$ a $30 \%$, o qual representa situações em que o períneo não atinge a dilatação adequada, evitando dessa forma uma possível laceração (KÄMPF C e DIAS RB, 2018). Todavia, na prática a episiotomia é realizada em até $94 \%$ dos partos vaginais, sendo, no sistema público brasileiro, um dos procedimentos cirúrgicos de maior incidência (DENGO VAR, 2016). A equipe de saúde declara à paciente que a episiotomia é um procedimento que irá prevenir lacerações graves, preservar a função sexual e reduzir a incontinência urinária e fecal. Alega-se, também, que em recém-nascidos reduz a ocorrência de asfixia, retardo mental e possível hemorragia cerebral (CARNIEL F, et al., 2019).

No Brasil, essa prática cirúrgica é a exceção em que não se faz necessário o consentimento prévio da mulher (KÄMPF C e DIAS RB, 2018). Nesse sentido, sabe-se que situações em que não ocorrem comunicação e devida explicação a respeito do procedimento, assim como cenários em que não há 
autorização pela paciente para a realização da episiotomia, configuram-se não apenas violência obstétrica, mas violência contra a autonomia da mulher, "integridade pessoal" e contra a ética profissional (GARRETT CA, et al., 2016).

Dessa forma, a falta de orientação em relação ao procedimento é considerada também como mutilação genital e que resulta em traumas à mulher, sejam esses de aspecto psicológico ou emocional. Como consequência imediata, pode-se ter dor acentuada no momento do parto e, quanto aos traumas do pósparto, pode acarretar prejuízos no desempenho sexual e dispareunia (CARNIEL F, et al., 2019). Em se tratando de sequelas físicas, a longo prazo, a episiotomia aumenta o risco para dor, assim como, pode causar incontinência urinária e fecal, prolapso de órgãos, como também aumenta o risco para laceração perineal de terceiro e quarto grau, infecções e hemorragia (PRIETO LNT, et al., 2017; GARRETT CA, et al., 2016).

Evidencia-se que o parto deveria ser lembrado como um momento especial pelas parturientes, mas isso se torna improvável quando estas se percebem em uma situação que the causa dor, sofrimento físico e mental e que comprometa sua dignidade. Dessa forma, o objetivo do presente estudo é analisar, por meio da revisão literária, em que momento a episiotomia deixa de ser um benefício e se torna uma violência obstétrica.

\section{REVISÃO BIBLIOGRÁFICA}

\section{Parto vaginal e a prática da episiotomia}

Nos últimos anos, o amparo ao parto e ao nascimento vivenciou uma alteração considerável, modificando a prática domiciliar para a institucionalizada (SALES JL, et al., 2020). Dessa maneira, o parto deixou de ter um caráter natural e passou a ser visto cada vez mais como um processo complexo, o qual necessita de intervenções e medicalização em demasia. Isto, ainda que não tenha sido o objetivo inicial, levou a mediações que muitas vezes geram consequências negativas, tanto para a parturiente quanto para o recém-nascido (SÁEZ ZA, et al., 2019).

O parto apropriado, seja vaginal ou cesárea, é aquele que garante a saúde da mãe e da criança. Tal via de parto deve ser escolhida de acordo com a vontade da mulher, contanto que seja possível e que a decisão tenha sido consciente. Além disso, os pré nnatalistas precisam explicar qual melhor via de parto, incentivando a mulher a escolher procedimentos que favoreçam o trabalho de parto e afastando práticas que possam causar algum dano ao processo fisiológico (SALES JL, et al., 2020).

O parto vaginal ideal é aquele em que a dilatação ocorre de forma gradual, simultaneamente com a descida fetal progressiva, acomodando no canal vaginal. Quando ocorre esse processo, as lacerações vaginais espontâneas tendem a não ocorrer ou serem em graus leves, já as lacerações mais profundas podem ser formadas durante a descida rápida, pela passagem da cabeça do feto e dilatação da vagina. Elas são classificadas em quatro graus: o primeiro envolve mucosa e pele perineal, o segundo estende ao músculo perineal, o terceiro chega até o músculo do esfíncter anal e o quarto se prolonga à mucosa anal (GÜN I, et al., 2016).

A episiotomia é um corte realizado na área perineal envolvendo pele, músculo e mucosa vaginal, de preferência, à $45^{\circ}$ mediolateral direita e que tem como objetivo ampliar a abertura vaginal, durante a fase expulsiva do parto (CARNIEL F, et al., 2019; TURMO M, et al., 2014). Embora existam diferentes tipos de incisão, como a mediana, a mediolateral e a lateral, com o passar dos anos tornou-se evidente que a lateralização da episiotomia concede melhores resultados para a gestante em virtude da diminuição da incidência de lesões obstétricas do esfíncter anal (NECESALOVA P, et al., 2016).

Tal técnica é utilizada com a justificativa de reduzir as lacerações durante o parto, preservando a musculatura do períneo e a função sexual materna, além de prevenir a incontinência urinária e fecal. Como benefícios para o recém-nascido são citados a diminuição de traumatismos cranianos, hemorragia cerebral, asfixia e retardo mental (VILLELA JP, et al., 2016). 
Entretanto, estudos atuais elucidaram o mito de que o parto é melhor assistido quando envolve intervenções médicas como a episiotomia, visto que tal incisão angaria para as gestantes malefícios tanto psicológicos quanto físicos, evidenciando dessa forma que se não realizada de forma seletiva, deixa de ter seu caráter profilático, passando a ser considerada como uma violência à autonomia da gestante (VILLELA JP, et al., 2016).

\section{Episiotomia: uso rotineiro versus seletivo}

O processo parturitivo possui alguns riscos característicos desse momento, como a possibilidade de ocorrer lacerações perineais, as quais podem se acentuar até o grau IV, podendo gerar a falta de controle do esfíncter anal e urinário e dispareunia. Dessa forma, as gestantes, além de estarem impelidas a tais riscos intrínsecos ao parto, também estão sujeitas a lesões consequentes à medicalização do nascimento, como as advindas da prática da episiotomia (SANTOS LM, et al., 2018). Isto posto, através de estudos nas últimas décadas, muitos cientistas chegaram a um consenso de que tal incisura, quando feita de forma seletiva, ainda que diminua a quantidade de procedimentos feitos, consegue preservar a saúde da região perineal de muitas gestantes (SCHANTZ C, et al., 2015).

Para aqueles que defendem o uso cotidiano da episiotomia, as principais razões são acerca da possível prevenção de lesões perineais, bem como a prevenção da perda do controle esfincteriano anal e urinário. Além disso, em razão de reduzir o tempo total do parto, em tese, acarretaria um maior amparo ao recémnascido (SÁEZ ZA, et al., 2019). Ademais, outro fator que difundiu tal prática rotineiramente foi a crença de que, em primíparas, essa incisura atuaria como prevenção à dor sentida durante a parição, além de permitir um retorno mais veloz ao estado perineal original anterior à gestação (CLESSE C, et al., 2019).

Por outro lado, há indivíduos que defendem que a prática da episiotomia deve ser utilizada apenas em casos específicos, como em situações em que o recém nascido pesa mais que quatro quilos; quando a mulher encontra-se em sua primeira gestação; quando o período expulsivo do parto tem longa duração; em partos vaginais cirúrgicos e quando há dificuldade da passagem do ombro da criança pelo canal vaginal, situação mais conhecida como distócia de ombro (JUNIOR MD e JÚNIOR RP, 2016).

Por anos a episiotomia foi amplamente utilizada por obstetras com o objetivo de facilitar o nascimento. Entretanto hoje, com o avanço da ciência, existem questionamentos sobre sua indicação. Tal procedimento que antes era tido como necessário para a conclusão do parto, atualmente deve ser usado apenas quando há riscos de lacerações perineais graves (graus III e IV). Após a influência da Medicina Baseada em Evidências, mais profissionais repensaram os benefícios da episiotomia e, com isso, observa-se um declínio dessa prática, principalmente em países desenvolvidos (CLESSE C, et al., 2019). Porém, um dos motivos que contribuem para a propagação desse ato é que ele ainda é ensinado em universidades e hospitais, tornando-se um hábito para profissionais da saúde, que regularmente não questionam sua necessidade, mesmo diante de tantas comprovações científicas (PRIETO LNT, et al., 2017). Esse fenômeno é reforçado pelo modelo de assistência ao parto no Brasil, que é centrado no médico e na atenção hospitalar (OLIVEIRA VJ e PENNA CMM, 2017).

Há, ainda, médicos que defendem essa prática através do discurso de que atualmente exista uma banalização do termo "violência obstétrica" pela mídia e de que o seu papel como detentor do saber seja suficiente para tomar as decisões adequadas à gestante. Deste modo, caso questionado pelo acompanhante, o profissional sente-se confrontado por considerar sua verdade como absoluta, o que reforça a ideia de uma relação por vezes conflituosa entre obstetra e gestante (OLIVEIRA VJ e PENNA CMM, 2017).

Ademais, no entendimento de tais apoiadores, a episiotomia, como qualquer outro procedimento médico, tem suas indicações e exige uma técnica cirúrgica e, em virtude disso, não é conveniente considerá-la como violência se feita corretamente (JUNIOR MDC e JÚNIOR RP, 2016).

Os profissionais contrários ao uso rotineiro da episiotomia justificam que é fundamental modificar a dinâmica atual, em que os recursos tecnológicos se sobrepõem ao conhecimento científico. Tal mudança objetiva evitar o emprego excessivo e contínuo de técnicas em circunstâncias que sejam dispensáveis. 
Dessa forma, acredita-se que obstetras bem preparados compreendem a fisiologia do parto e, por esse motivo, realizam menos intervenções, apenas quando se faz necessário (KÄMPF C e DIAS RB, 2018). Além disso, foi implementado no Brasil em 2000, o Programa de Humanização do Pré-natal e Nascimento (PHPN) como forma de assegurar à gestante um tratamento digno e desestimular procedimentos desnecessários (SALES JL, et al., 2020).

\section{Episiotomia no Brasil e fatores determinantes de uma boa prática}

O Brasil vem sofrendo um decréscimo na taxa de realização de episiotomia, transitando de $94 \%$ nos anos 2000 para 54\% em 2014. Entretanto, ainda que tenha havido melhorias consideráveis, o número atual ainda é alarmante, sobretudo quando comparado a outros países. No cenário nacional, a prática foi melhor aplicada por profissionais cujas idades são inferiores a 40 anos, por profissionais com formação acadêmica finalizada há menos de 10 anos e/ou que trabalham em cargos públicos e em cidades que são capitais nacionais. Essas relações são justificadas pela percepção de que indivíduos mais novos e com formações acadêmicas mais recentes estão sujeitos, durante a graduação, a evidências científicas mais atualizadas, bem como são mais adeptos a mudanças no meio científico, quando comparados com profissionais acima de 40 anos. $O$ fato de a ocupação ser em capitais e em cargos públicos facilita a incorporação de manuais mais atualizados, bem como os profissionais promoverem uma educação continuada mais efetiva (CUNHA CMP, et al., 2019).

Nesse ínterim, relatou-se que, na teoria, muitos médicos estão cientes dos benefícios da episiotomia realizada seletivamente, todavia na prática isso ainda não é aplicado, uma vez que mesmo com o conhecimento exposto aos profissionais, a prática rotineira ainda ocorre. Por fim, constatou-se também que muitos profissionais possuem o imaginário de que o corpo da mulher é fundamentalmente defeituoso, o que justificaria a medicalização excessiva durante o processo parturitivo (CUNHA CMP, et al., 2019).

\section{As consequências da episiotomia}

É evidente que o trauma perineal tem papel de destaque dentre as implicações clínicas da episiotomia, o qual é responsável por provocar dor nas puérperas, e, portanto, prejudica suas atividades cotidianas, como por exemplo, deambular e sentar, além das necessidades fisiológicas (SILVA AMN, et al., 2019). Embora muitos profissionais fundamentem o uso da episiotomia de forma rotineira com base na crença de que ela reduz os riscos de lacerações perineais, estudos evidenciaram que entre seus efeitos adversos estão lacerações importantes e mais graves, como as de grau III e IV (SAÉZ ZA, et al., 2019), e do mesmo modo, a incontinência fecal e urinária também são sequelas a longo prazo decorrentes da episiotomia de rotina (GUN I, et al., 2016).

A tensão realizada no procedimento de episiorrafia, que consiste em suturar a episiotomia, aumenta em 1,7 vezes a chance de que a mulher apresente edema, pois ocorre uma maior demanda de reação inflamatória pelo organismo, o que ocasiona um acúmulo de fluídos no local da sutura. Ademais, à episiorrafia também podem estar associados outros sinais e sintomas como equimose, infecção local e hematoma (SANTOS LM, et al., 2018).

Comumente, mulheres que foram submetidas à episiotomia no parto vaginal tendem a retomar a prática sexual de forma mais lenta se comparado a mulheres que não passaram por tal experiência (MOURA TR, et al., 2018), uma vez que a episiotomia pode desencadear dispareunia, sentimentos de vergonha e temor em retornar à prática sexual. É notório que a aparência estética da região genital se modifica consideravelmente, podendo provocar grande indignação na mulher, visto que toda a circunstância poderia ter sido evitada por meio de um esclarecimento prévio e consequente respeito à sua vontade (VILLELA JP, et al., 2016).

\section{Episiotomia como violência obstétrica}

Ao observar puérperas que passaram pela experiência da episiotomia, inúmeros relatos são similares e as principais queixas encontradas são de mulheres que não receberam orientações adequadas sobre esse procedimento e que foram excluídas da tomada de decisão no momento do parto. Logo, é evidente que existe uma falha durante o pré-natal, em que gestantes não são informadas sobre procedimentos que 
podem ser necessários, suas indicações, consequências e sobre a autonomia da mulher (VILLELA JP, et al., 2016). Muitas vezes, o pouco conhecimento da gestante sobre a episiotomia é passado por um familiar ou amiga que teve uma experiência prévia com essa técnica, ou até mesmo em momentos onde o profissional de saúde se dispôs a explicar sobre tal, porém com certa distorção de sua indicação (FIGUEIREDO G, et al., 2015).

Outro fato discutido é o Consentimento Informado da gestante sobre qualquer procedimento realizado nela. Caso a mulher não tenha esclarecimento prévio sobre a episiotomia, essa prática é enquadrada como violência obstétrica com abuso de ação intervencionista. Essa é a principal causa de revolta nas parturientes, visto que grande parte dos profissionais responsáveis pela saúde da mulher age com menosprezo diante deste momento importante da gestação (GARRETT CA, et al., 2016). O uso abusivo e sem indicações da episiotomia tornou-se um desrespeito aos direitos das mulheres e, dessa forma, gera repercussões para a parturiente tais como a falta de protagonismo durante o trabalho de parto devido à perda do direito de escolha no instante da tomada de decisões (VILLELA JP, et al., 2016).

No pós-parto, o impacto decorrente de uma episiotomia sem consentimento tem sequelas maiores que as marcas físicas. Os principais sentimentos das puérperas são de violação e medo. Nesse ponto, há ainda outras falhas dos profissionais de saúde, que muitas vezes continuam a negligenciar informações que poderiam instruir as puérperas e permitir que elas vivenciassem de modo mais humanizado o pós-parto (VILLELA JP, et al., 2016). Diante disso, a violência obstétrica não é apenas pela forma como a episiotomia é empregada, mas pela postura do obstetra frente à gestante, com discursos ofensivos na sala de parto, relação de hierarquia em que o médico é detentor do saber e hostilidade diante dos desejos da mulher (OLIVEIRA VJ e PENNA CMM, 2017).

Percebe-se, portanto, uma persistência significativa do uso rotineiro da episiotomia na prática médica mesmo frente às comprovações científicas e influências da Medicina Baseada em Evidências e da OMS sobre a necessidade do seu uso limitado. A análise do conjunto de estudos demonstra que a episiotomia é considerada como um ato de violência obstétrica quando realizada sem o esclarecimento e consentimento da gestante e com alegações médicas que não condizem com a realidade da parturiente. Observa-se que a autonomia dos obstetras sobre as mulheres diante das decisões a serem tomadas no parto e a constância dessa técnica cirúrgica de forma habitual estão fortemente associadas à formação remota dos profissionais. Quanto aos médicos formados nos últimos 10 anos, os estudos revisados apontam uma maior adaptação às mudanças científicas para a realização da episiotomia, demonstrando um avanço nessa área (CUNHA CMP, et al., 2019).

\section{CONSIDERAÇÕES FINAIS}

Nesse cenário, é evidente a iminência da capacitação médica, sobretudo para os profissionais de formação acadêmica mais antiga, objetivando renovar os conhecimentos técnico-científicos para recuperar a condição não patológica do momento de criação e valorizar a proposta de humanização do parto. Somando-se a isto, visto a carência de conhecimentos das parturientes sobre seus direitos, é necessário ampliar o olhar da gestante e de seus familiares sobre seu poder de decisão na assistência ao parto, por meio de campanhas sociais informativas. Tal medida é essencial para alcançar uma postura respeitosa por parte da equipe médica em relação aos desejos e valores no ciclo gravídico. Recomendam-se novos estudos sobre métodos alternativos à episiotomia que não abrangem ideias intervencionistas e abuso medicamentoso no parto. Assim sendo, reforça-se que a episiotomia deve se limitar aos casos específicos, como proposto por seu criador Fielding Ould.

\section{REFERÊNCIAS}

1. CARNIEL F, et al. Episiotomia de rotina: necessidade versus violência obstétrica. J. nurs. health, 2019; 9(2): e199204.

2. CARROLI G, MIGNINI L. Episiotomy for vaginal birth. Cochrane Database Syst Rev, 2009; (1):CD000081. 
3. CLESSE C, et al. Socio-historical evolution of the episiotomy practice: A literature review. Women \& Health, 2019; 59:7, 760-774.

4. CUNHA CMP, et al. Knowledge, Attitude and Practice of Brazilian Obstetricians Regarding Episiotomy. Rev Bras Ginecol Obstet, 2019;41:636-646.

5. DENGO VAR. A episiotomia na percepção de puérperas. Cogitare Enferm, 2016; 21(3): 01-08.

6. FIGUEIREDO G, et al. Episiotomy: perceptions from adolescent puerperae. Invest Educ Enferm, 2015; 33(2): 365373.

7. GARRETT CA, et al. O uso da episiotomia no sistema único de saúde brasileiro: a percepção das parturientes. Revista Saúde e Pesquisa, 2016; 9(3): 453-459.

8. GÜN I, et al. Long- and short-term complications of episiotomy. Turk J Obstet Gynecol, 2016; 13:144-8.

9. JUNIOR MD, JÚNIOR RP. Selective Episiotomy: Indications, Techinique, and Association with Severe Perineal Lacerations. Rev Bras Ginecol Obstet, 2016; 38:301-307.

10. KÄMPF C, DIAS RB. A episiotomia na visão da obstetrícia humanizada: reflexões a partir dos estudos sociais da ciência e tecnologia. História, Ciências, Saúde - Manguinhos, Rio de Janeiro, 2018; 25(4): 1155-1160.

11. MOURA TR, et al. Dispareunia relacionada à via de parto: uma revisão integrativa. Rev. Ciênc. Méd., 2018; 27(3):157-165.

12. NECESALOVA $P$, et al. Mediolateral versus lateral episiotomy and their effect on postpartum coital activity and dyspareunia rate 3 and 6 months postpartum. Sexual \& Reproductive Healthcare, 2016.

13. OLIVEIRA VJ, PENNA CMM. Discussing obstetric violence through the voices of women and health professionals. Texto Contexto Enferm, 2017; 26(2):e06500015.

14. PRIETO LNT, et al. A episiotomia de rotina é uma prática baseada em evidência? Revista CuidArte Enfermagem, 2017 jul-dez; $11(2)$ : 269-278.

15. SÁEZ ZA, et al. Estudio comparativo de la recuperación postparto en base a los Patrones de Marjory Gordon. Revista electrónica trimestral de Enfermería, 2019; 18(53): 183-214.

16. SALES JL, et al. Assistência ao parto em um hospital da baixada litorânea do Rio de Janeiro: desafios para um parto respeitoso. Rev Fun Care Online. 2020; 12: 108-114.

17. SANTOS LM, et al. Associação entre perineorrafia e problemas perineais, atividades habituais e necessidades fisiológicas afetadas. Rev Cuid, 2018; 9(2): 2233-2244.

18. SCHANTZ C, et al. Reasons for routine episiotomy: A mixed-methods study in a large maternity hospital in Phnom Penh, Cambodia. Reproductive Health Matters, 2015; 23(45):68-77.

19. SILVA AMN, et al. Characterization of pain resulting from perineal trauma in women with vaginal delivery. Br $\mathrm{J} P a i n$, 2018; 1(2):158-62.

20. TURMO M, et al. Cronificación del dolor tras episiotomía. Rev Esp Anestesiol Reanim. 2014.

21. VILLELA JP, et al. Episiotomia: sentimentos vivenciados pelas puérperas. Rev enferm UERJ, 2016; 24(5):e21882. 